\title{
Some new hypotheses on the mediation of loudness judgments'
}

WILLARD R. THURLOW AND LAWRENCE E. MELAMED

UNIVERSITY OF WISCONSIN

A new solution to the problem of relating equal-interval and ratio scales is proposed. It is shown that it is also possible, with the type of the ory proposed, to predict results of cross-modality matching experiments.

A persisting puzzle in psychophysical scaling is that the scales derived by equal-interval method are typically not related linearly to scales derived by ratio methods. However, when the scale based on judgment of equal differences is plotted against the logarithm of the ratio scale, often the resulting graph is not far from a straight line (Schneider \& Lane, 1963; Ekman \& Sjöberg, 1965). As Torgersen (1961) states the problem: "According to the subject's own judgments, stimuli separated by equal subjective intervals are also separated by approximately equal subjective ratios."

This result appears to be difficult to make sense of so long as one conceives of loudness scaling as a simple process of attaching numbers to an inferred inner magnitude corresponding to sensory input (Stevens, 1961). Some theorists have reacted to this problem by claiming that one of these scales is the more valid one; and some claim that the other type of scale is due to confusion or error on the part of the subject. Thus Garner (1954) has argued that presumed erroneous ratios used by subjects should be corrected from equal-interval data. Stevens and Guirao (1962) argue that category scale values can be understood as biased away from ratio scale values because of variability. (Recent results obtained by Schneider and Lane (1963) do not support the latter interpretation, however.) Stevens (1958) has criticized Helson's Adaptation Level Theory (See Helson, 1964)-which predicts category judgments by means of a formulation accepting Fechner's logarithmic law-because Helson's theory does not adequately predict the results of ratio scaling procedures. Stevens seems to imply quite clearly that results obtained from ratio-scaling procedures are the more valid.

The main purpose of this paper is to suggest that a solution to the problem exists if one assumes that the subject can make use of a common mediating response when asked to judge an interval or a ratio. (We assume that typical subjects would be forced to use a mediating response because they have never been trained to associate numbers directly with loudness.) The subject, for instance, might move his hand along an imagined scale from a setting of 1 in. to a setting of 2 in. Decibel changes which caused this amount of response change would be judged equal. On the other hand, this response change from 1 to 2 in. could be conceived of by the subject as a ratio of 2 to 1. Thus, ambiguity could arise. Data from the following experiment concerns the relation of a possible mediating response (distance of movement along a scale) to sound intensity. It is very important to note that we will use this data to clarify the nature of the theory we wish to present. It is not our purpose to predict the data from the theory.

\section{EXPERIMENT: DISTANCE RESPONSES TO CHANGES IN TONAL LOUDNESS}

Pairs of tones were presented to the subject every 5 sec. Each tone was $1000 \mathrm{cps}$ in frequency, $1 \mathrm{sec}$. in duration. There was an interval of $.10 \mathrm{sec}$. between tones. The first tone was a standard loudness $(11,31$, 41 , or $71 \mathrm{~dB}$ above average threshold), while the second was either the same intensity as the standard, $\pm 3 \mathrm{~dB}$, or $\pm 5 \mathrm{~dB}$ with respect to the standard. The 1000 cps stimulus was produced by an oscillator, then led through mixer, attenuators, switch, filter, and transformer to the subject's earphone. The subject listened in a separate sound-insulated room.

The subject moved a stylus down a groove with his left hand for a standard distance $(6 \mathrm{~cm})$ when the first tone of each pair sounded. For the second tone of each pair, he was instructed to try to move a stylus in his right hand (down a groove) a distance which was appropriate to the loudness. Thus, if the second tone was louder, the second movement would be appropriately larger than the movement made to the first, standard tone. If the second tone was softer, the second movement would be appropriately smaller than the movement made to the first, standard tone. The subject was blindfolded.

Each intensity standard was used on a different day. Order of standards was determined by a latin square. Eight subjects took part in the experiment, and made 18 judgments for each stimulus in each session.

Figure 1 shows the mean distance (in centimeters) moved, corresponding to decibel changes in tone intensity from the standard tones.

\section{"Ratio" problem}

\section{DISCUSSION}

Derivation of a "power law" for loudness. It is assumed that the subject who was asked to judge fractions or multiples of loudness would make use of a constant change in mediating response. A possible 


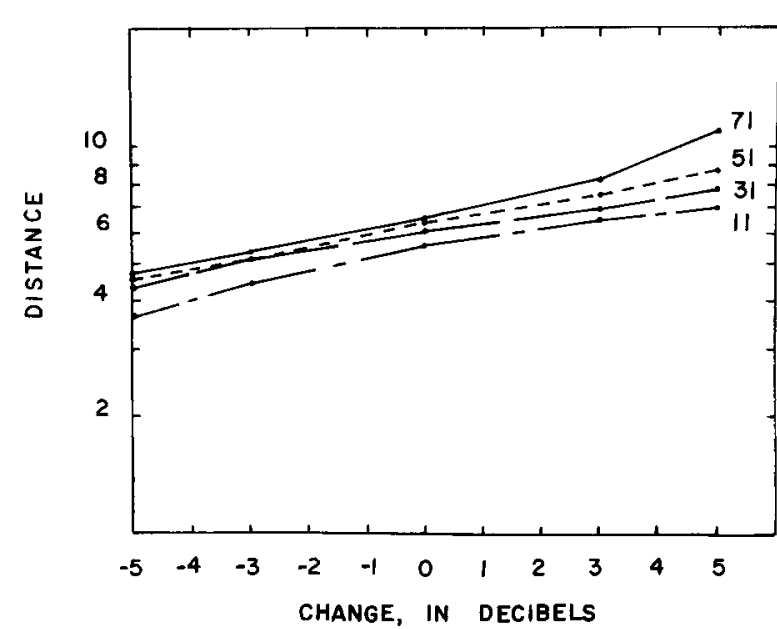

Fig. 1. Average distance moved in centimeters as a function of decibel change in stimulus. Standard loudnesses were at 11,31 51, and 71 decibels sensation level.

mediating response might be that used as the dependent variable in the experiment above. If the subject was imagining moving his hand along a scale, then he might judge that the loudness had doubled when his hand had moved from 6 units on the scale to 12 units on the scale. Extrapolating from our illustrative data, this would require a change in intensity of about $10 \mathrm{~dB}$, near the value Stevens chose as representing the intensity change required to produce a 2 to 1 change in loudness (Stevens, 1955). In applying ratio numbers to this criterion of a constant change in mediating response (from a constant mediating response made to the standard tone), the subject would generate a scale which would follow the power law; e.g., a ratio increase in loudness numbers would correspond to a ratio increase in sound pressure. From the relationship shown in Fig. 1-a linear relation between $\log$ response and log sound pressure-it can be seen that the final numerical scale would be the same whether the subject in a particular case adopted a criterion for his $2 / 1$ ratio of a change from 6 to 12 , 4 to 8 , or some other doubling.

It must be remembered, of course, that there are a number of other variables which will influence the final function obtained. For example, with the magnitude estimation technique, the sound-pressure level of the standard chosen, and the size of the number assigned to the standard have a very definite influence (Stevens, 1956; Hellman \& Zwislocki, 1961; Jones \& Woskow, 1966). Our argument concerns the determinant which is felt to be most important in producing a power function-the mediating response, and the way numbers are applied to this response.

When the magnitude estimation technique is used, an alternative hypothesis for what the subject is doing is as follows: It can be supposed that he is making a cross-modality match between an internal response due to tone and that of a measuring response (Attneave, 1962; Ekman, 1964). A power-law prediction has been obtained from this theoretical approach. It is possible that both hypotheses may be correct: some subjects may follow the matching strategy, while other subjects may follow the strategy we outlined in a preceding paragraph.

\section{Cross-modality matching.}

The theory which we have presented needs to be developed further in order to explain some other important results obtained with loudness judgments. Previous investigators have found it possible to predict the results of matching the loudness of a tone with the force of hand-grip by means of the ratio of the exponents of the power functions for numerical (ratio) scales of loudness and force (Stevens, Mack, \& Stevens, 1960; Stevens, 1961; Attneave, 1962). How can this prediction be accomplished, and still be related to the type of theory discussed in this paper?

Assume that for force of handgrip there is a response, $R_{F}$, which is related to the force applied on

a dynamometer, $F$ by: $R_{F}=k_{1} \log \frac{F}{F_{0}}$. (This "response" could represent input from the sensory system, or a transformation of this input in a higher center of the nervous system; it has the status of an intervening variable.)

If we have a function for loudness $R_{L}=k_{2} \log \frac{P}{P O}$ (where $\mathrm{p}$ refers to sound pressure), then it is assumed that matching occurs when $R_{F}=R_{L}$. Thus, $k_{1} \log \frac{F}{F_{0}}=$ $k_{2} \log \frac{P}{P_{0}} ;$ then $\log \frac{F}{F_{0}}=\frac{k_{2}}{k_{1}} \log \frac{P}{P_{0}}$. Attneave (1962) and Ekman $(1964,1965)$ have previously suggested this possibility.

Can the slope, $\frac{k_{2}}{\bar{k}_{1}}$, be predicted (by our theory) from numerical scales of force of handgrip, and of loudness, derived by a "fractionation" procedure? Assume that a constant change in $R_{L}$ produces (or is equated to) an equivalent change in an internal response $R_{m}$, which is associated with a change in an external standard measuring response " $M$ " by the equation: $R_{m}=a \log \frac{M}{M_{0}}$.

This " $M$ " measuring (mediating) response might be one like that portrayed in Fig. 1. (Note that a crucial assumption in our theory is that the subject can apply the criterion of a constant change in standard measuring response, $M$, over and over again, even though the intensity level of the standard stimulus changes; see Fig. 1.) 
Now assume that a certain ratio change in sound pressure $\frac{P_{1}}{P_{0}}$ produces this change in the standard measuring response, $M$, which is interpreted as a $2 / 1$ increase (along the $M$ scale). The "sensation," $S$, would grow by the formula: $S_{L}=2 y$, where $y$ is the number of times the ratio was applied. Let $y=\log _{\frac{p}{p_{0}}} \frac{\left(\frac{p}{p_{0}}\right)}{\text { (where }}$ $p$ is the pressure finally arrived at after the ratio $\frac{P}{P O}$ has been applied y times). Then $\log _{\frac{P}{1}} S_{L}=y \log \frac{P_{1}}{P O}$.

$$
\text { PO PO }
$$

Let $\log _{p_{1}} 2=C$. Then $\log _{\frac{p}{p_{1}}} S_{L}=C \log _{p_{1}}\left(\frac{p}{P_{0}}\right)$, or $S_{L}=$ $\left(\frac{P}{P_{0}}\right)^{C}$. This represents an equation for a numerical "ratio" scale of loudness.

If $R_{F}=k_{1} \log \frac{F}{F_{0}}$ and $R_{L}=k_{2} \log \frac{P}{P_{D}}$, then in order for a change in handgrip force ratio to produce the same change in internal response $R_{F}$ as in $R_{L}$ (produced by the change $\frac{P_{1}}{P_{0}}$ in sound pressure), $F$ would $\underset{k_{2}}{\text { have }}$ to change by the ratio $\left(\frac{P_{1}}{P_{0}}\right) \bar{k}_{1}$, since $k_{1} \log \left(\frac{P_{1}}{P_{0}}\right)^{k_{1}}=$ $\left(\frac{k_{2}}{k_{1}}\right) k_{1} \log \frac{P_{1}}{P_{0}}=k_{2} \log \frac{P_{1}}{P_{0}} \cdot$ But if force of handgrip changes by the ratio $\left(\frac{p}{p_{0}}\right)^{\bar{k}}$, for each change in the unit measuring response $M$ (which is interpreted by the subject as a doubling of "sensory" magnitude), then $S_{F}=$ $\left(\frac{\mathrm{P}}{\mathrm{P}_{0}}\right) c^{\mathrm{k}_{\mathrm{k}}} \mathrm{k}_{2}$, where $S_{F}$ represents a numerical scale of force.

Thus the ratio of the exponents for the numerical (ratio) scales of loudness and force (C divided by

c $\frac{k_{1}}{k_{2}}$ predicts the slope of the power function obtained by cross-modality matching $\left(\begin{array}{l}k_{2} \\ k_{1}\end{array}\right)$. A similar line of reasoning can be applied to other cross-modality matches with loudness (Stevens, 1966).

If one assumes that the numerical "power law" is derived not from the successive use of the same measuring response increment (as we have above), but from matching an internal response (for example, $\mathbf{R}_{\mathrm{L}}$, due to tone) with an internal response caused by a measuring response such as our " $M$ "' (Attneave, 1962; Ekman, 1964), it is possible to readily derive prediction of slopes of cross-modality matches of tone to force of handgrip from slopes of numerical "magnitude" scales for loudness and force of handgrip.
Assume, as before: $R_{L}=k_{2} \log \frac{P}{P_{0}}, R_{F}=k_{1} \log \frac{F}{F_{0}}$, and $R_{m}=a \log \frac{M}{M_{0}}$. Loudness is measured by a numerical scale of "M" when: a $\log \frac{M}{M_{0}}=k_{2} \log \frac{P}{P_{0}}$. The slope is $k_{2}$. Force of handgrip is measured by a numerical scale of " $M$ " when: a $\log \frac{M}{\bar{M}_{0}}=k_{1} \log \frac{F}{F_{0}}$; the slope will be $\frac{k_{1}}{o}$. If now loudness is matched to (and measured by) force of handgrip, $k_{1} \log \frac{F}{F_{0}}=k_{2} \log \frac{P}{P O}$. The slope is $\frac{k_{2}}{k_{1}}$. This slope is predicted by the ratio of the slopes of numerical scales for loudness and force of handgrip: $\frac{k_{2}}{k_{1}}=\frac{k_{2}}{a} / \frac{k_{1}}{a}$. It is clear that predicted slope for the cross-modality matches is the same from each of the two theories. ${ }^{2}$

Although we have formulated equations for internal events as logarithmic equations, it would be possible to formulate them as "power-law" equations. This formulation, however, would require an internal mechanism for equating ratios of magnitudes.

Tests of these predictions should be made with individual subjects, in order that the same measuring response and hence the same criterion for "doubling of sensation" would be used in the different experiments. Stevens and Guirao have shown that exponents for the loudness ratio scale may vary considerably from one individual to another (Stevens \& Guirao, 1964).

\section{"Equal inferval" problem}

Now we return to Fig. 1 to illustrate the way in which the theory would explain judgment of equal intervals. The theory assumes that in judging equal intervals the subject can apply the same increment in measuring response, $M$, over and over again, as a criterion of equality of interval. The (mediating) measuring response used by the subject could be movement along a scale, as in Fig. 1. The subject would adjust two stimulus intervals to equality by adjusting the measuring response increment to equality.

In Fig. 1, if there were not the tendency for subjects to respond with a larger movement to louder ranges of tones, the decibel increase necessary to produce a constant increment in distance of movement (over that to the standard tone) would be nearly constant at different sensation levels. Thus (without this "error" tendency) if a constant movement increment is the criterion for an "equal sensation difference," then we would expect to get a linear relation between sound pressure in decibels and numbers assigned to the equal intervals of an equal interval scale. Garner's data on equal interval judgments shows an almost linear relation to decibels (Garner; 1954). 
Of course, there is slight curvature in the equalinterval data functions. (Note that we do not refer to the derived equal interval scale.) One of the causes of the curvature could be the tendency, obvious in Fig. 1, of subjects to give a somewhat larger response to higher level stimuli. (A Friedman analysis of variance by ranks shows significant differences between intensity conditions beyond the .01 level.) If a response increase from the standard response to some fixed magnitude is the subject's criterion for an equal difference in sensation, then the decibel increment necessary to produce this response increase would be less at higher decibel levels. Garner's data show this type of curvature (Garner, 1954). There are other minor biasing factors in the equal-interval judging situation which can lead to slight changes in curvature (Garner, 1954). In category scaling there are even more biasing factors which would make a simple prediction difficult (Tabory \& Thurlow, 1959; Luce \& Galanter, 1963). A complete theory will obviously have to take these factors into account in a quantitative way (Parducci, 1965). Nevertheless, there is an approximately linear relation between category judgments and decibels-a relation which we feel could be understood mainly in terms of the use by the subject of essentially a constant change in a mediating response as a criterion of a constant sensation difference.

\section{References}

Attneave, F. Perception and related areas. In S. Koch (Ed.), Psychology: A study of a science, Vol. 4: Biologically-oriented fields: Their place in psychology and in biological science. New York, 1962. Pp. 619-659.

Ekman, G. Is the power law a special case of Fechner's law? Percept. mot. Skills, 1964, 19, 730.

Ekman, G., \& Sjoberg, L. Scaling. In P. R. Farnsworth (Ed.), Annual review of psychology. Palo Alto, Cal.: Ann. Rev. Inc., 1965. Pp. 451-474.

Gamer, W. R. A technique and a scale for loudness measurement. J. Acoust. Soc. Amer., 1954, 26, 73-88.

Hellman, R. P., \& Zwislocki, J. Some factors affecting the estimation of loudness. J. Acoust. Soc. Amer., 1961, 33, 687-694.

Helson, H. Adaptation-level theory. New York: Harper \& Row, 1964.

Jones, F. N., \& Woskow, M. J. Some effects of context on the slope in magnitude estimation. J.exp. Psychol, 1966, 71, 177180.
Luce, R. D., \& Galanter, E. Psychophysical scaling. In R. D. Luce, R. R. Bush \& E. Galanter (Eds.), Handbook of mathematical psychology. New York: Wiley, 1963.

Parducci, A. Category judgment: a range-frequency model. Psychol. Rev., 1965, 72, 407-418.

Schneider, B., \& Lane, H. Ratio scales, category scales and variability in the production of loudness and softness. J. Acoust. Soc. Amer., 1963, 35, 1953-1961.

Stevens, J. C., \& Guirao, M. Individual loudness functions. $J$. Acuust. Soc. Amer, 1964, 36, 2210-2213.

Stevens, J. C., Mack, J. D., \& Stevens, S. S. Growth of sensation on seven continua as measured by force of handgrip. $J$. exp. Psychol, 1960, 59, 60-67.

Stevens, S. S. The measurement of loudness. $J$. Acoust. soc. Amer., 1955, 27, 815-829.

Stevens, S. S. Adaptation level vs. the relativity of judgment. Amer. J. Psychol., 1958, 71, 633-646.

Stevens, S. S. The psychophysics of sensory function. In W. A. Rosenblith (Ed.), Sensory communication. Cambridge, Mass. and New York: Technology Press, and Wiley, 1961. Pp. 586-606.

Stevens, S. S. Matching functions between loudness and ten other continus. Percept. \& Psychophys., 1966, 1, 5-8.

Stevens, S. S., \& Guirao, M. Loudness, reciprocality and partition scales. J. Acoust. Soc. Amer., 1962, 34, 1466-1471.

Tabory, L., \& Thurlow, W. Judgments of the loudness of a series of tones with two different range expectancies. J. gen. Psychol., $1959,60,167-172$.

Torgersen, W. S. Distances and ratios in psychophysical scaling. Acta Psychologica, Amsterdam, 1961, 19, 201-205.

\section{Notes}

1. This research was supported by a grant from the University Research Committee of the University of Wisconsin from funds provided by the Alumni Research Foundation. The first author is responsible for the theoretical analysis. The second author collected the data used for illustration of the theory.

2. Another (perhaps intuitively simpler) method of explaining by our theory the prediction of slope of cross-matching force of handgrip with loudness is as follows: If the subject assigns a ratio of numbers to each constant increase in response $M$ (as for example a ratio increase in number of 2 to 1 - to correspond to an increase in $M$ response from 6 to 12 , for an increase of $10 \mathrm{~dB}$ in sound pressure), he will generate a numerical loudness scale with the same slope as he would by cross-matching loudness with the $M$ scale. Similarly, applying the constant increment in measuring response, $M$, over and over, he will generate a numerical scale for force of handgrip with the same slope as he would by cross-matching force of handgrip to the $M$ scale. The slope for cross-matching force of handgrip with loudness is then predicted (as indicated in the preceding paragraph)ty the ratio of the slopes of the numerical scales for loudness and force of handgrip.

(Accepted for publication Decenber 4, 1966.) 